\title{
Early molecular correlates of adverse events following yellow fever vaccination
}

\author{
Candice Y.Y. Chan, ${ }^{1,2}$ Kuan Rong Chan, ${ }^{1}$ Camillus J.H. Chua, ${ }^{3}$ Sharifah nur Hazirah, ${ }^{3}$ Sujoy Chosh, ${ }^{4}$ \\ Eng Eong Ooi, ${ }^{1,5,6,7}$ and Jenny G. Low ${ }^{1,2}$ \\ 'Program in Emerging Infectious Diseases, Duke-NUS Medical School, Singapore. ²Department of Infectious Diseases, \\ Singapore General Hospital, Singapore. ${ }^{3}$ SingHealth Translational Immunology and Inflammation Centre (STIIC), \\ Singapore. ${ }^{4}$ Centre for Computational Biology, Duke-NUS Medical School, Singapore. ${ }^{5}$ Department of Microbiology and \\ Immunology, Yong Loo Lin School of Medicine, National University of Singapore, Singapore. ${ }^{6}$ Saw Swee Hock School \\ of Public Health, National University of Singapore, Singapore. IInterdisciplinary Research Group in Infectious Diseases, \\ Singapore-MIT Alliance for Research \& Technology (SMART), Singapore.
}

The innate immune response shapes the development of adaptive immunity following infections and vaccination. However, it can also induce symptoms such as fever and myalgia, leading to the possibility that the molecular basis of immunogenicity and reactogenicity of vaccination are inseparably linked. To test this possibility, we used the yellow fever live-attenuated vaccine (YFLAV) as a model to study the molecular correlates of reactogenicity or adverse events (AEs). We analyzed the outcome of 68 adults who completed a YFLAV clinical trial, of which $43(63.2 \%)$ reported systemic AEs. Through whole-genome profiling of blood collected before and after YFLAV dosing, we observed that activation of innate immune genes at day 1, but not day 3 after vaccination, was directly correlated with AEs. These findings contrast with the gene expression profile at day 3 that we and others have previously shown to be correlated with immunogenicity. We conclude that although the innate immune response is a double-edged sword, its expression that induces AEs is temporally distinct from that which engenders robust immunity. The use of genomic profiling thus provides molecular insights into the biology of AEs that potentially forms a basis for the development of safer vaccines.

Authorship note: C.Y.Y. Chan and K.R. Chan contributed equally to this work.

Conflict of interest: The authors have declared that no conflict of interest exists.

Submitted: July 5, 2017

Accepted: August 29, 2017

Published: October 5, 2017

Reference information: JCI Insight. 2017;2(19):e96031. https://doi.org/10.1172/jici. insight.96031.

\section{Introduction}

Vaccines have played a foundational role in preventing infectious diseases. Smallpox and polio, which have collectively devastated millions of lives for centuries, have now been eradicated and eliminated in most parts of the world, respectively, through the widespread implementation of effective vaccines (1). Several other childhood or congenital diseases, such as measles, mumps and rubella, have also been controlled through vaccination programs. Despite the success of vaccination, however, there is an increasing mistrust in vaccines stemming from the adverse events (AEs) following vaccination (2). While much of the fear is indeed misguided, there remains a paucity of information on the molecular basis of AEs. This contrasts with the efforts that have already been expanded to understand the molecular basis of immunogenicity, findings from which have provided insights on how vaccines could be designed to elicit improved immunogenicity. We posit that a detailed understanding of the molecular processes that lead to AEs would provide a molecular basis to develop vaccines with fewer AEs and a more objective approach to evaluating vaccine safety.

The molecular basis of immunogenicity has been studied using peripheral blood samples collected from individuals vaccinated with the yellow fever live-attenuated vaccine (YFLAV) (3-6). This vaccine is both safe and highly immunogenic, with long-term immunity engendered from a single dose (7). Previous studies have consistently identified the innate immune response, particularly expression of genes in the Tolllike receptor and type I IFN pathways at days 3 to 7 after vaccination, as predictive of the adaptive immune response to vaccination (3-8). These findings are consistent with the known role of the innate immune response in activating the adaptive immune response, the memory of which confers long-term immunity. However, the innate immune response is partly proinflammatory, which is responsible for symptoms such as fever, myalgia, and headache typically encountered in acute febrile illness (9). That the degree of innate 
Enrollment

Allocation

\begin{tabular}{|l|}
\hline Analysis \\
for \\
systemic \\
AEs \\
\hline
\end{tabular}

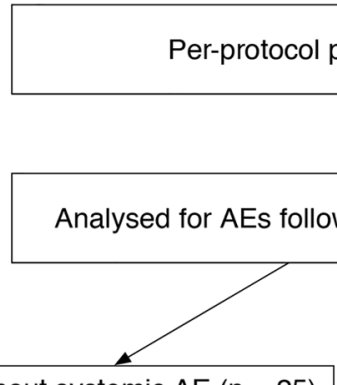

Subjects without systemic AE $(n=25)$
Assessed for eligibility $(n=196)$

Excluded $(n=112)$

- Dengue IgG positive $(n=72)$

- Abnormal physical examination /

biochemistry $(n=26)$

- Withdrew consent before

randomisation $(n=14)$

\section{Randomised $(\mathrm{n}=84)$}

Allocated to intervention $(\mathrm{n}=84)$

-Received JE and/or YF vaccination ( $n=74)$

-Did not receive allocated intervention $(n=10)$

(10 subjects withdrew voluntarily and did not

receive their YF vaccination at the stipulated time)

- Voluntarily withdrew from the trial before 1 month

post YF vaccination follow-up $(n=4)$

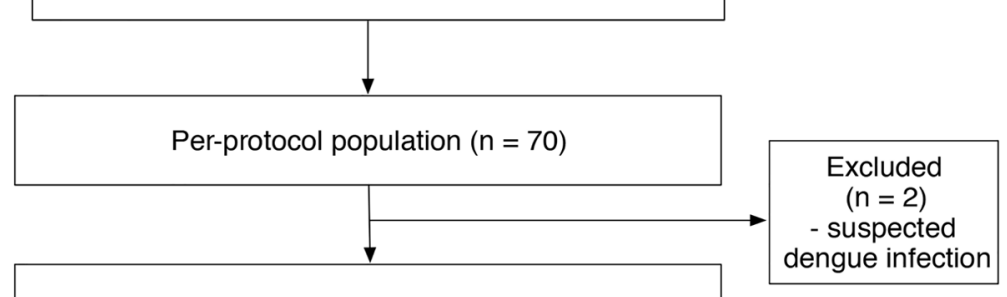
dengue infection

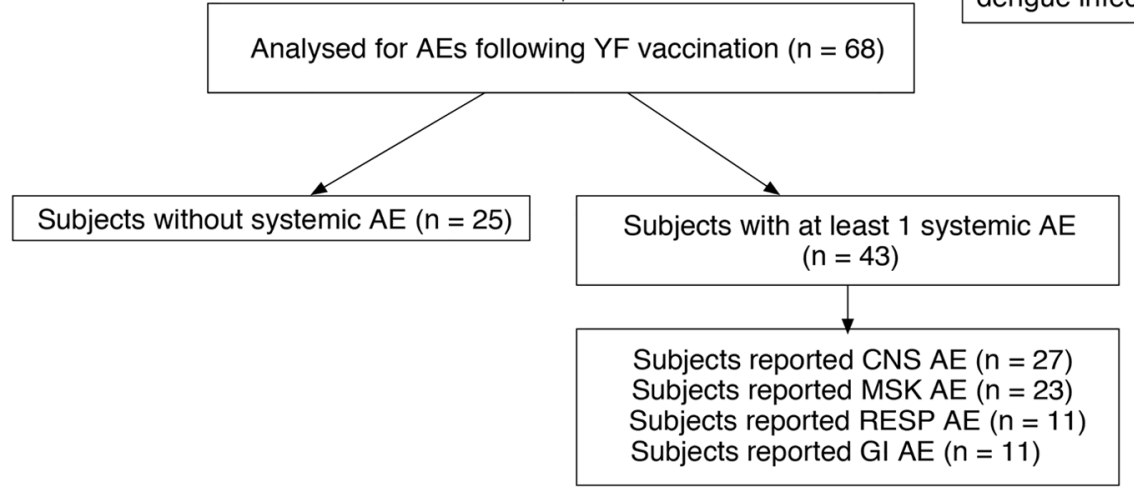

Figure 1. Consort diagram of the study. Subjects were vaccinated with yellow fever live-attenuated vaccine (YFLAV) with or without history of prior Japanese encephalitis (JE) vaccination. Subjects were followed up for development of adverse events (AEs) for 1 month. Each systemic AE was subcategorized by system organ class: CNS, musculoskeletal (MSK), respiratory (RESP), and gastrointestinal (GI). Blood was sampled for antibody levels, viremia, and gene expression at stipulated time points (see main text for details)

immune response is directly correlated with the robustness of adaptive immune response suggests that potent vaccines are inherently reactogenic; the innate immune response could drive both vaccine immunogenicity and AEs (10). If this is true, then immunogenicity and reactogenicity are a molecularly intertwined yin-yang of vaccination; AEs are undesired but necessary collateral effects of potent vaccines.

Here, we report an exploratory analysis of a randomized, open-label clinical trial on YFLAV to test the hypothesis that the correlates of immunogenicity also correlate with AEs. We identify the molecular signatures of systemic AEs and show that, although the innate immune pathways are also associated with the development of postvaccination AEs, their correlation with AEs and immunogenicity are temporally distinct.

\section{Results}

Characteristics of subjects and YFLAV AEs. The original trial protocol explored the effect of cross-reactive antibodies on YFLAV immunogenicity (5). Figure 1 shows the number of subjects enrolled. Of the 68 subjects that completed the study per protocol, 43 (63.2\%) reported systemic AEs. No significant difference in age, body mass index, gender, and ethnicity were found between those with or without AEs (Table 1). Overall, our published $\mathrm{AE}$ rates were comparable to the previously reported AE rates following YFLAV administration $(21 \%-72 \%)$, depending on the study design (11-15). Using the distribution of fever onset as a guide, as it has been shown to be a significant AE in those who received YFLAV compared with placebo (11), we divided the AEs into immediate or delayed based on whether the AE onset occurred before or after 24 hours after vaccination (Figure 2, A-C). Of the 43 subjects who reported AEs, 26 subjects reported only delayed AEs while 16 reported immediate AEs that fully resolved before new-onset delayed AEs. Only 1 subject reported immediate $\mathrm{AE}$ without onset of any new $\mathrm{AE}$ after the first 24 hours after vaccination. Hence, 17 subjects had immediate AEs, while 42 had delayed AEs (Figure 2A). The distribution of the types of immediate and delayed AEs is shown in Figure 2B, as well as Table 2. The delayed AEs have a median onset of 6 days after vaccination (Figure 2C and Table 3), and lasted for a median of 1 day (Supplemental Figure 1; supplemental material available online with this article; https://doi.org/10.1172/jci.insight.96031DS1). The immediate AEs were limited to mostly fatigue that lasted also for a median of 1 day. Given the larger numbers with delayed AEs and to avoid confounding our findings with multiple analyses of the 16 subjects with both immediate and delayed AEs, we thus focused our analysis on identifying the correlates of delayed AEs. All AEs were coded 
Table 1. Demographics of our trial subjects

\begin{tabular}{|c|c|c|c|c|c|c|c|}
\hline Subjects ( $n=68$ ) & No AE & All SYS AE & Local AE & CNS AE & MSK AE & RESP AE & GI AE \\
\hline Subjects (\%) & $25(36.8)$ & $43(63.2)$ & $26(38.2)$ & 27 (39.7) & $23(33.8)$ & $11(16.2)$ & $11(16.2)$ \\
\hline $\begin{array}{l}\text { Age (in years) } \\
\text { Median (range) }\end{array}$ & $28(22-47)$ & $29(21-50)$ & $28(22-46)$ & $29(22-46)$ & $29(22-50)$ & $29(23-40)$ & $27(21-32)$ \\
\hline \multicolumn{8}{|l|}{ Gender } \\
\hline Male (\%) & $9(36.0)$ & $25(58.1)$ & $11(42.3)$ & 14 (51.9) & $13(56.5)$ & $7(63.6)$ & $5(45.4)$ \\
\hline \multicolumn{8}{|l|}{ Ethnicity $n(\%)$} \\
\hline Indian & $1(4.0)$ & $3(7.0)$ & $0(0.0)$ & $2(7.4)$ & $1(4.3)$ & $0(0)$ & $0(0)$ \\
\hline Others ${ }^{A}$ & $2(8.0)$ & $4(9.3)$ & $2(7.7)$ & $3(11.1)$ & $2(8.7)$ & $1(9.1)$ & $2(18.2)$ \\
\hline \multicolumn{8}{|l|}{$\mathrm{BMI} \mathrm{kg} / \mathrm{m}^{2}$} \\
\hline Mean $( \pm \mathrm{SD})$ & $22.8( \pm 3.9)$ & $22.0( \pm 3.0)$ & $21.5( \pm 2.9)$ & $21.9( \pm 2.8)$ & $21.7( \pm 2.8)$ & $21.8( \pm 1.9)$ & $21.2( \pm 2.1)$ \\
\hline
\end{tabular}

Age, gender, ethnicity, and BMI of the subjects that have completed the trial and were analyzed for adverse events (AEs) attributed to yellow fever liveattenuated vaccine (YFLAV) ( $n=68)$. AEs were segregated by system organ class: systemic (SYS), CNS, musculoskeletal (MSK), respiratory (RESP), and gastrointestinal (GI). ${ }^{A}$ Others: Caucasian, Chinese-Japanese.

according to the Common Terminology Criteria for Adverse Events (CTCAE), version 4.0 (16). Subjects with systemic AEs were classified as possible, probable, or definite based on clinical evaluation by the investigators during study follow-ups. Of the 43 subjects, 29 had possible and 14 had probable systemic AEs (Supplemental Table 1). None of the systemic AEs were classified as definite; all documented definite AEs were localized reactions. Likewise, none of the subjects in our cohort developed severe neurotropic or viscerotropic disease.

Anti-YF antibody titers and YFLAV viremia levels in subjects. To determine if immunogenicity was intricately linked with AEs, we compared the YF-neutralizing antibody titer at 1 and 6 months after vaccination in those that experienced delayed AEs with those without any AEs (Figure 2D). No significant difference between these groups was observed at either postvaccination time point. Likewise, no difference in YFLAV viremia levels (Figure 2E) at day 3 or 7 was observed between subjects with or without AEs; day 7 viremia had previously been shown to be directly correlated with YF-neutralizing antibody titers at 1 month after vaccination (5).

Systemic AEs are associated with upregulation of innate immune gene sets at day 1 after vaccination. Whole-blood genome changes following vaccination were measured using microarray. Total RNA was extracted from whole blood of 26 subjects (18 with delayed AEs, 8 without AEs) that were collected immediately before YFLAV administration (day 0), as well as at days 1 and 3 after YFLAV. This data set was previously analyzed to determine the correlates of immunogenicity (5). To identify the gene expression changes associated with AEs, we performed gene-set enrichment analysis (GSEA) on the microarray data (17). A total of 14 gene sets in blood obtained at day 1 after vaccination were enriched in the delayed AE group versus the non-AE group (Supplemental Table 2). Gene sets with the highest normalized enrichment scores (NES) included multiple Toll-like and NOD-like pattern recognition receptors (PRRs), innate immune system, and IFN signaling (Figure $3 \mathrm{~A}$ ). Specific genes within these pathways were upregulated between days 0 and 1 in those who reported AEs. In contrast, these same genes were mostly downregulated in those who did not report AEs (Figure 3B and Supplemental Figure 2, A-F). These gene sets remain significantly enriched even after excluding the 6 subjects who reported both immediate and delayed AEs from analysis (Supplemental Figure 3).

To determine if the expression of these genes segregated according to the classification of AEs, we analyzed those with probable $(n=7)$ and possible $(n=11)$ AEs separately, and compared each to those without AEs. Despite the smaller number of subjects, comparison between probable AEs with no AE identified differentially expressed pathways at statistically significant levels (Supplemental Figure 4A). Comparison of possible AEs with no AE, on the other hand, failed to identify any pathway that reached statistical significance (Supplemental Figure 4B), although a heatmap of the genes belonging to Toll-like and NOD-like PRRs as well as the IFN signaling pathways shows distinct trends in subjects with systemic AEs compared with those without symptoms (Supplemental Figure 4C). This analysis thus identifies further support for the notion that expression of genes in the innate immune pathways is associated with development of systemic AEs. 
A

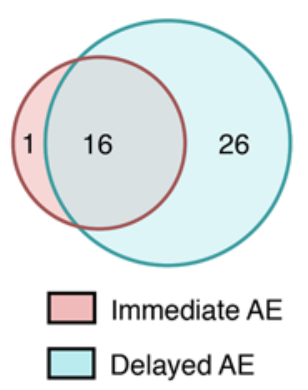

B

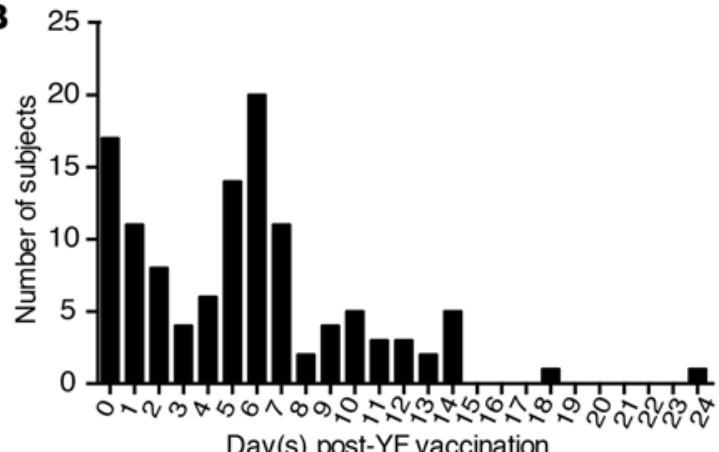

c
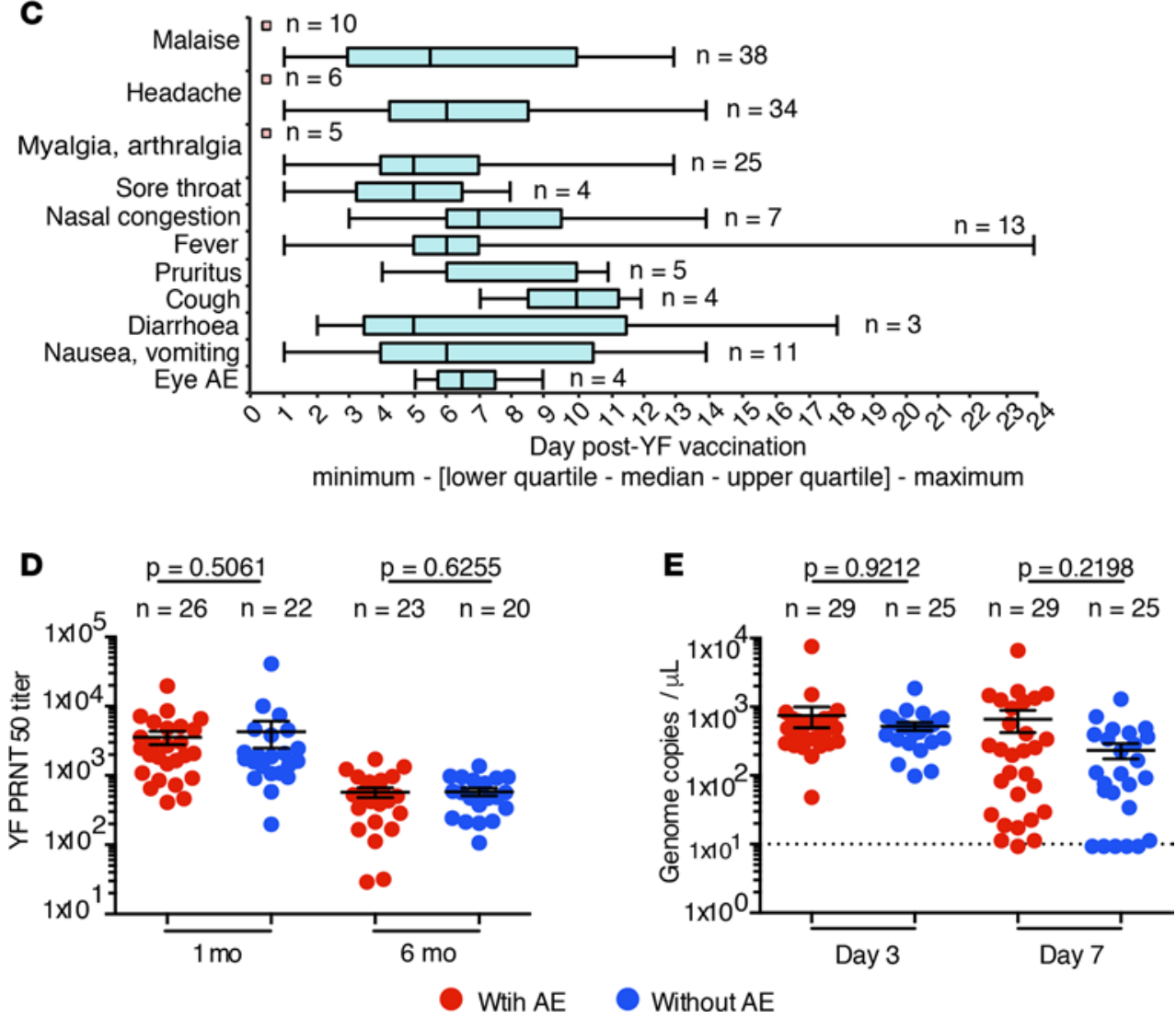

Figure 2. Delayed systemic AEs are not correlated with YF immunogenicity or vaccine viremia. (A) Venn diagram showing number of subjects who reported only immediate adverse events (AEs) that occurred less than 24 hours after yellow fever live-attenuated vaccine (YFLAV) administration $(n=1)$, only delayed AEs that occurred more than 24 hours after vaccination $(n=26)$, and immediate $A E s$ that fully resolved before new-onset delayed AEs $(n=16)$. (B) Histogram showing the number of subjects with reported AEs by day of onset after YF vaccination. (C) Box-and-whisker plot showing the day of onset of specific symptoms after $Y F$ vaccination (the line within the box indicates the median, the end of the box shows the 25th and 75th percentile, and ends of the whiskers are minimum and maximum). Red bars represent immediate AEs reported 24 hours or less after $Y F$ vaccination. Blue bars represent delayed $A E s$ reported more than 24 hours after $Y F$ vaccination. Only events reported more than once are shown. $n=$ number of events. (D) YF-neutralizing antibody titers at 1 month after vaccination in subjects with delayed $A E$ (red) or without $A E$ (blue) as measured by plaque neutralization reduction test (PRNT). Data are expressed as the PRNT titer that neutralized $50 \%$ of the viral inoculum (PRNT ${ }_{50}$ ). (E) YFLAV RNA levels in peripheral blood measured by qPCR at days 3 and 7 after vaccination in subjects with delayed $A E s$ or without AEs. In $\mathbf{D}$ and $\mathbf{E}, P$ values were obtained by 2-tailed Mann-Whitney test, and mean $\pm \mathrm{SEM}$ is shown. Sample sizes are depicted in the figure. Dotted line depicts limit of detection.

To validate the microarray findings, we used the nCounter assay (Nanostring Technologies), as it offers advantages including high sensitivity, reproducibility, and multiplexity using limited amounts of clinical material (18). This analysis identified the same sets of genes that were shown to be differentially regulated by microarray (Supplemental Figure 5A and Supplemental Table 3). Likewise, analysis of another set of samples not previously analyzed by microarray also identified the same sets of genes as being differentially expressed in those with AEs compared with those without (Supplemental Figure 5, B-H). The expression of several of these genes also directly correlated with the number of reported symptoms by the subjects; the higher the expression of these genes, the more symptoms were reported by the subjects (Figure 3C and Supplemental Table 4).

In contrast to the day 1 data, no gene sets were enriched in blood of subjects with delayed AEs taken at day 3 after vaccination compared with those who did not report any symptom (Figure 3D and Supplemental Figure 6A). This finding was consistent even after the subjects who reported both immediate and delayed AEs were removed from analysis (Supplemental Figure 6, B-D). Collectively, these observations indicate that differential expression of innate immune genes at day 1 after vaccination is associated with the development of systemic AEs. 
Table 2. Characteristics of systemic AEs attributed to YFLAV administration

\begin{tabular}{|c|c|c|c|c|}
\hline \multirow[t]{4}{*}{ Systemic } & Fever & $13(19.1)$ & $0(0)$ & $13(100)$ \\
\hline & Pruritus & $3(4.4)$ & $0(0)$ & $5(100)$ \\
\hline & Swollen lymph node & $1(1.5)$ & $0(0)$ & $1(100)$ \\
\hline & Rashes & $1(1.5)$ & $0(0)$ & $1(100)$ \\
\hline CNS & Pre-syncope & $1(1.5)$ & $0(0)$ & $1(100)$ \\
\hline \multirow[t]{3}{*}{ MSK } & Myalgia & $24(35.3)$ & $5(17.2)$ & $24(82.8)$ \\
\hline & Arthralgia & $1(1.5)$ & $0(0)$ & $1(100)$ \\
\hline & Neck stiffness & $1(1.5)$ & $0(0)$ & $1(100)$ \\
\hline Respiratory & Sore throat & $5(7.4)$ & $1(25)$ & $4(75)$ \\
\hline Gastrointestinal & Vomiting & $2(2.9)$ & $0(0)$ & $2(100)$ \\
\hline \multirow[t]{2}{*}{ Eye } & Orbital edema & $1(1.5)$ & $0(0)$ & $1(100)$ \\
\hline & Eye pain/redness & $3(4.4)$ & $0(0)$ & $3(100)$ \\
\hline
\end{tabular}

Gene sets associated with MSK and CNS AEs. Next, we examined the AEs by organ system, namely the musculoskeletal (MSK) and CNS, with the transcriptome obtained from postvaccination day 1 blood samples. GSEA identified gene sets that were differentially regulated in those with delayed MSK or CNS AEs (Figure 4, A and B, and Supplemental Tables 5-7). As expected, the pathways associated with both MSK and CNS AEs were PRRs, cytokines, and inflammatory signaling processes (Figure 4C and Supplemental Table 5). These findings suggest that innate immune, cytokine, and inflammatory responses modulate nociceptive pathways and thus contribute to hyperalgesia $(19,20)$

The gene sets that were uniquely enriched in vaccine recipients with MSK AEs included adenosine diphosphate (ADP) signaling via purinergic receptors $P 2 R Y 1$ and $P 2 R Y 12$, as well as metabolism of sphingolipids, glycerophospholipids, and glycerosphingolipids (Figure 4, D and G, and Supplemental Table 6). Interestingly, the genes in the P2RY1 and P2RY12 pathways are predominantly G protein-coupled receptors and signaling components belonging to the family of $\mathrm{P} 2 \mathrm{Y}$ receptors. These receptors are widely expressed in neuronal and neuroglial cells as well as peripheral leukocytes and are known to play important roles in eliciting proinflammatory responses (21). They have previously been shown to be involved in the initiation and modulation of visceral, cutaneous, and MSK neuropathic as well as inflammatory pain following trauma and infection (22). They have also been associated with myalgic encephalomyelitis (23-26). In contrast, genes related to DNA synthesis and cell cycle regulation were specifically downregulated in those with MSK AEs (Figure 4, E and H, and Supplemental Table 6).

The top upregulated gene sets that were unique to those with delayed CNS AEs were signaling by stem cell factor (SCF) and semaphorin 4D (SEMA4D) signaling (Figure 4, F and I, and Supplemental Table 7). SCF binding to the tyrosine kinase receptor c-kit mediates numerous biological functions in hematopoietic cells and leukocytes that regulate hematopoiesis, erythropoiesis, inflammation, and activation of both innate and adaptive immune functions (27). SCF signaling in neurons is also known to play an important role in regulating pain sensitivity and pathogenesis of headache $(28,29)$. Interestingly, semaphorins were first identified as regulators of axonal growth, and subsequently shown to function in antigen-presenting cells and $\mathrm{T}$ cell stimulation (30). The association between semaphorin, SCF expression and headache suggests a vestigial neuroimmune interaction that remains to be fully defined. 
A

TOLL receptor cascades NOD1 2 signaling pathway Innate immune system IFN signaling

Thrombin signalling through PARS Signaling by ILS TRAF6 mediated IRF7 activation TAK1 mediates $\mathrm{p} 38$ MAPK activation TLR7 89 induction of NFKB \& MAPK ADP signalling through P2RY12.

C
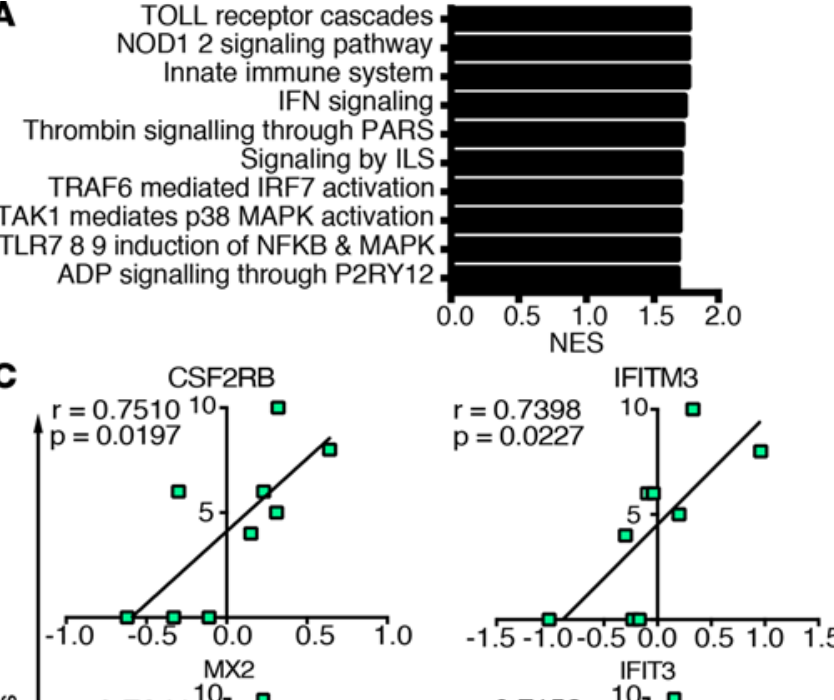

IFIT3

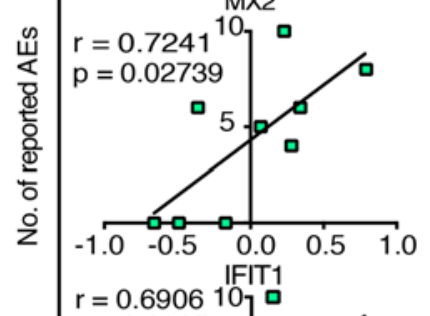

$\mathrm{p}=0.0395$

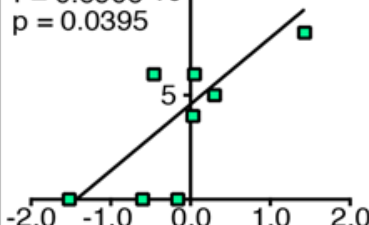

$r=0.7152$

$\mathrm{p}=0.03031$

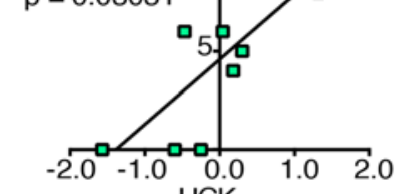

$r=0.6733$ HCK

$\mathrm{p}=0.0468$
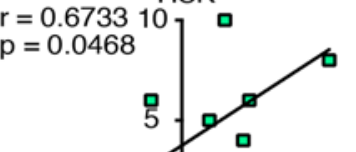

Log2 Fold Change (Day 1 / day 0)

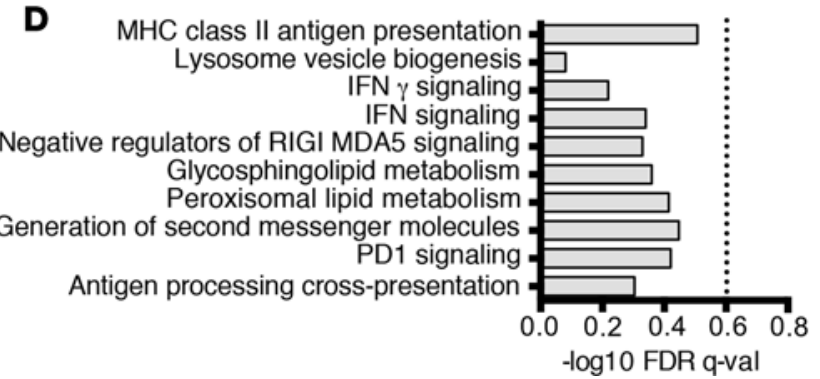

B

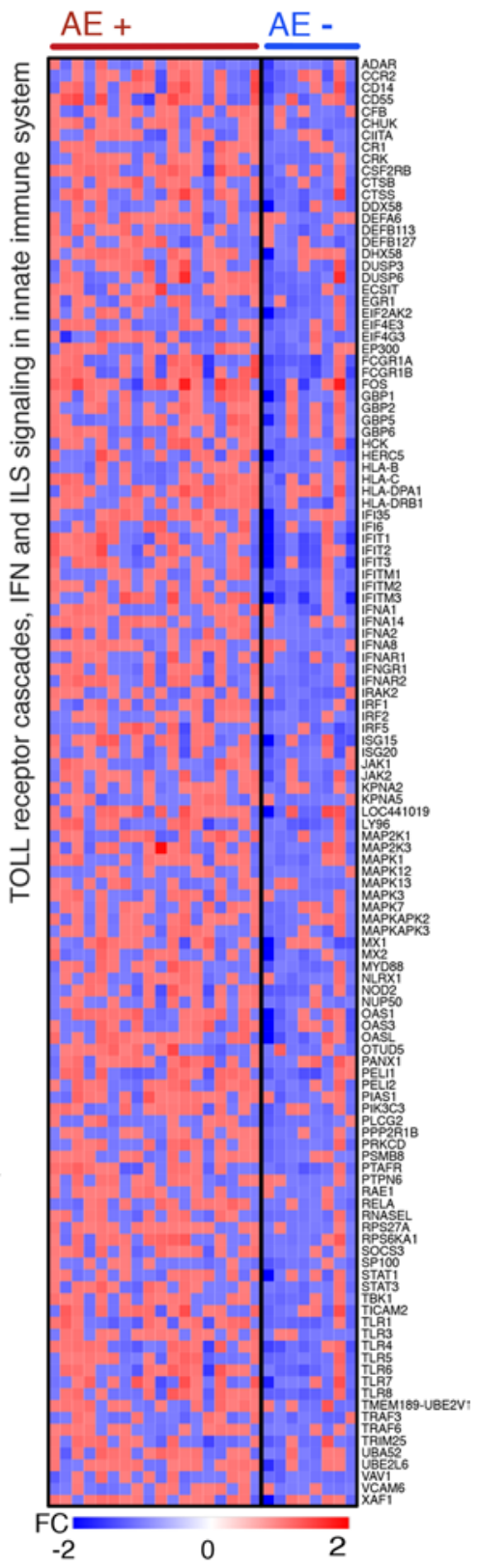

Figure 3. Whole-genome expression reveals significant differences in innate immune signaling pathways in those with delayed AEs. (A) Top 10 enriched gene sets identified by gene set enrichment analysis (CSEA) from venous blood microarray data in subjects with delayed adverse events (AEs) ( $n=18$ ) compared with subjects without AEs $(n=8)$ at day 1 after yellow fever (YF) vaccination, ranked by normalized enrichment scores (NES), with FDR $q$ values less than 0.25 . (B) Heatmap of the microarray data showing fold changes observed at day 1 versus day 0 , for genes in the Toll-like receptor cascades, IFN, and interleukins (ILS) signaling. (C) Pearson's correlation ( $r$ ) of log-transformed fold change (day 1 versus day 0) of genes CSF2RB, IFITM3, MX2, IFIT3, IFIT1, and HCK, measured using nCounter assay. All 9 subjects shown here were independent of the data set from the microarray analysis. $P$ values indicate significance of the slope. (D) Top 10 enriched gene sets from venous blood microarray data ranked by NES at day 3 after vaccination. Dotted line represents the cut-off FDR $q$ value of 0.25 .

We also took the opportunity to examine if any of the differentially expressed genes in our study overlapped with genes identified in autistic spectrum disorders. Cross-checking with the list of 261 curated human autism risk genes (Supplemental Table 8) from AutDB revealed no overlaps with those enriched in our subjects (31).

\section{Discussion}

YF vaccine has been used to identify the molecular correlates of immunogenicity. Induction of the innate immune and proinflammatory responses at days 3 to 7 after vaccination has been identified as correlates of the neutralizing-antibody response (3-6). In addition, we have also shown that a longer duration of viremia 
Table 3. Summary of local and systemic AEs attributed to YFLAV

\begin{tabular}{|c|c|c|c|c|c|}
\hline Subjects ( $n=68)$ & All SYS AE & CNS AE & MSK AE & RESP AE & GI AE \\
\hline No. of subjects with $\geq 1 \mathrm{AE}(\%)$ & $43(63.2)$ & $27(39.7)$ & $23(33.8)$ & $11(16.2)$ & $11(16.2)$ \\
\hline Total systemic AEs & 176 & 42 & 31 & 16 & 15 \\
\hline Immediate (\%) & $24(13.6)$ & $6(14.3)$ & $5(16.1)$ & $2(12.5)$ & $1(6.7)$ \\
\hline \multicolumn{6}{|l|}{ Onset ${ }^{A}$ of all $A E s$} \\
\hline Median (IQR) day & $5(2-7)$ & $5(2-7)$ & $5(2-6)$ & $7(4-9)$ & $6(3-11)$ \\
\hline \multicolumn{6}{|l|}{ Severity of AEs } \\
\hline Mild (\%) & $137(77.8)$ & $30(71.4)$ & $23(74.2)$ & $14(87.5)$ & $13(86.7)$ \\
\hline Moderate (\%) & $29(16.5)$ & $8(19.0)$ & $7(22.6)$ & $1(6.25)$ & $0(0)$ \\
\hline Severe (\%) & $6(3.4)$ & $3(7.1)$ & $1(3.2)$ & $0(0)$ & $1(6.7)$ \\
\hline Unclassified (\%) & $4(2.3)$ & $1(2.4)$ & $0(0)$ & $1(6.25)$ & $1(6.7)$ \\
\hline
\end{tabular}

Each systemic adverse event (AE) was subcategorized by system organ class: systemic (SYS), CNS, gastrointestinal (GI), musculoskeletal (MSK), and respiratory (RESP). ${ }^{\text {AD }}$ ay of onset after yellow fever live-attenuated vaccine (YFLAV) administration.

resulted in higher neutralizing-antibody levels following vaccination; viremia at day 7 but not day 3 after vaccination positively correlated with YF-neutralizing antibody titers (5). Our findings from this study add to this body of knowledge by demonstrating that the correlates of immunogenicity of YFLAV were not associated with delayed AEs. Instead, we show that an earlier activation of innate immune and proinflammatory genes, at day 1 after vaccination, drove the onset of delayed systemic AEs, the median time of which was at day 6 after vaccination (Supplemental Figure 7). Furthermore, the induction of innate immune response was significantly associated with probable AEs instead of possible AEs, despite a smaller number of subjects in this stratified analysis. The lack of association between the innate immune response at day 3 after vaccination and systemic AE is further supported by the observed lack of significant difference in neutralizingantibody titers against YF virus between those with and those without AEs; the innate immune response at day 3 but not day 1 correlated with YF-virus-neutralizing antibody titers (5). These findings thus suggest that the molecular determinants of immunogenicity and reactogenicity are not intricately linked.

Besides the correlation with systemic AEs, expression of specific sets of genes at day 1 after vaccination also appear to be associated with commonly reported symptoms, namely myalgia and headache. Many of these gene sets, such as the purinergic receptors and semaphorins, appear to play regulatory roles in CNS function as well as immune response. Our findings thus support the notion that the nervous and immune systems have evolved common signaling and cell-cell communication pathways (32). It would be interesting to explore the commonalities in development and evolution of these 2 systems and whether these shared pathways represent vestiges of a common progenitor.

The lack of association between viremia levels, either at day 3 or day 7 after vaccination, with delayed AEs is interesting. This finding is in contrast to previous studies on dengue where viremia levels appear to be correlated with clinical outcome of infection $(33,34)$. The rationale for these discrepant findings is unclear. The subjects in our study received a constant dose of YFLAV. Whether they develop AEs or not could thus be due to genetic heterogeneity in our subjects. Such genetic heterogeneity could also play a role in influencing the outcome of wild-type infection. However, wild-type infection is caused by inoculum from mosquito vectors that may not inject a constant dose of virus into the human host. Different strains of virus may replicate to higher titers in the mosquito salivary gland and there may also be variation in the amount of saliva secreted by individual mosquitoes during blood meal. All of these factors could influence the viral load in the inoculum that also shapes whether the infected individual develops an asymptomatic or symptomatic infection. 
A

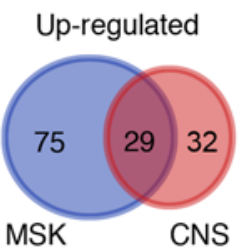

B

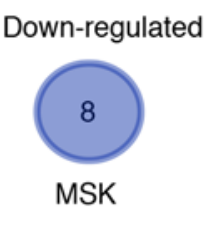

C $\quad=\mathrm{MSK}=\mathrm{CNS}$

IL1 signaling

Toll receptor cascades

Recycling pathway of $\mathrm{L} 1$ Signalling to RAS.

Activated TLR4 signalling

Activated TAK1 mediates p38 MAPK activation

TRAF6 mediated NFkB activation-

NfKB and MAPK activation mediated by TLR4-

Signalling to ERKS -

NLR signaling pathways-

D

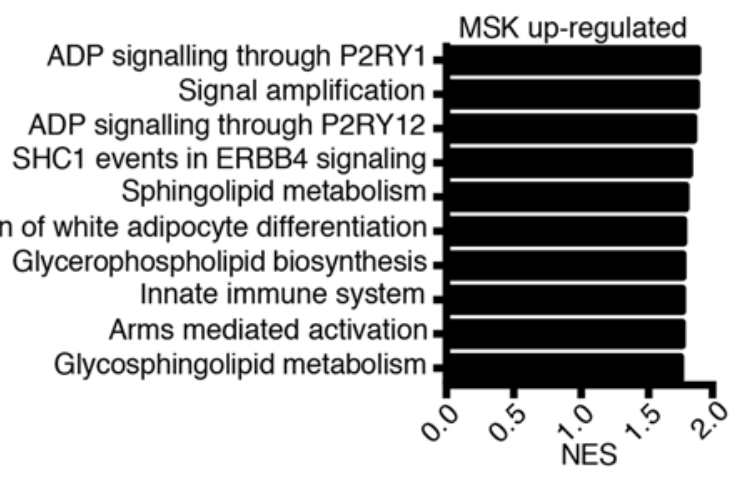

E

Transcriptional regulation of white adipocyte differentiation Innate immune system Arms mediated activation Glycosphingolipid metabolism

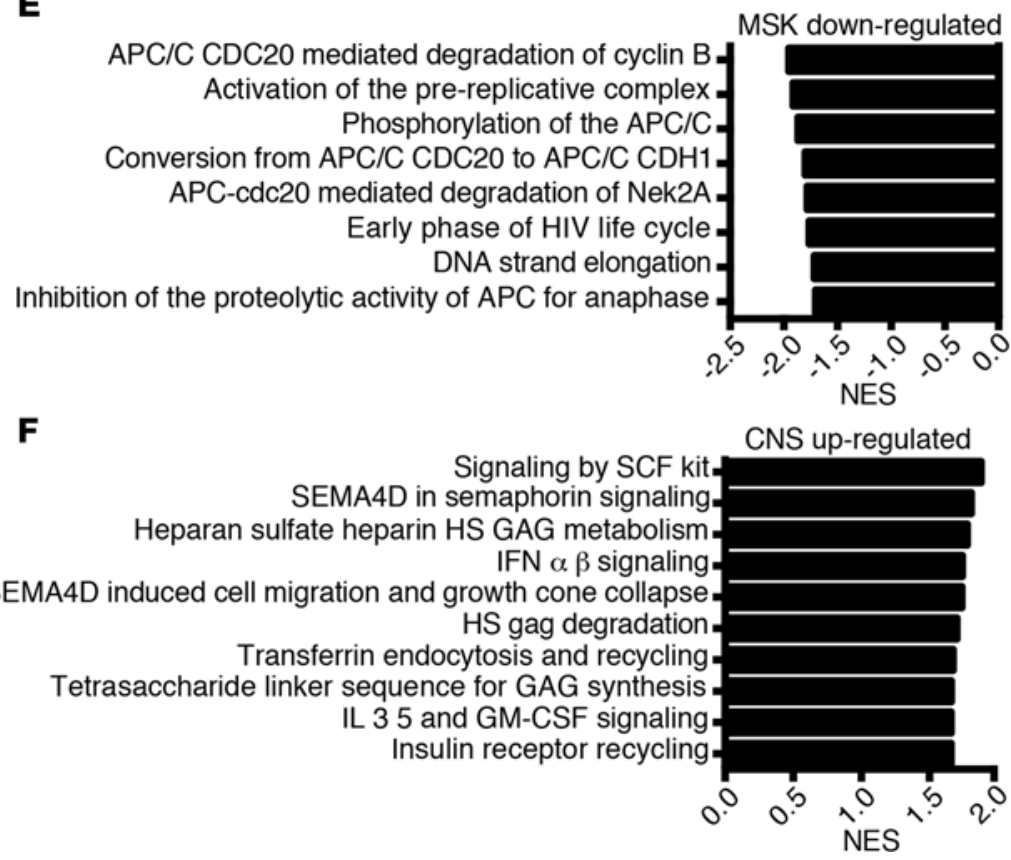

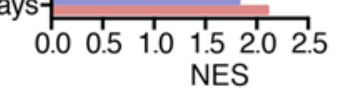

G ADP signaling, signal amplification

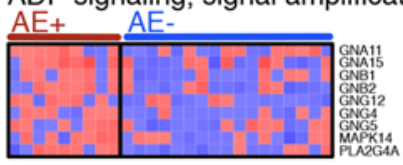

Sphingolipid metabolism

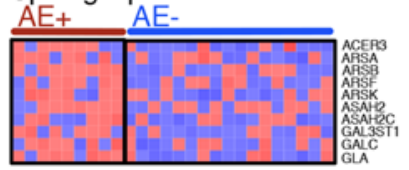

H APC/C regulation of cell cycle

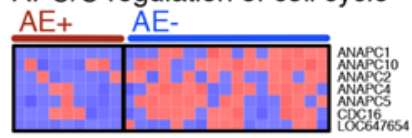

Activation of the pre-replicative complex

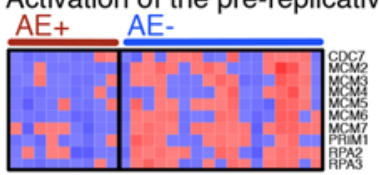

I Signaling by SCF kit

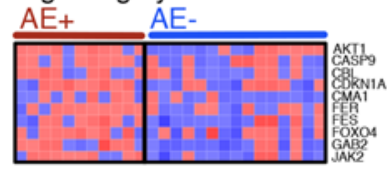

SEMA4D in semaphorin signaling
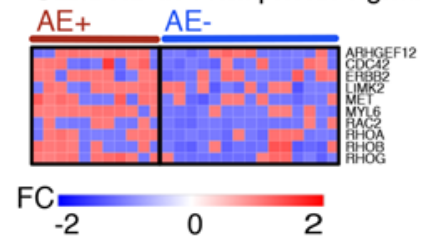

Figure 4. Genes that are differentially expressed in vaccinees with MSK and CNS AEs. The number of (A) upregulated gene sets, or (B) downregulated gene sets identified by GSEA (FDR $q$ values $<0.25$ ) from venous blood microarray data in subjects with delayed musculoskeletal (MSK) ( $n=9$ ) or CNS ( $n$ = 11) symptoms, at day 1 after vaccination. Overlap indicates the number of significantly enriched gene sets shared between subjects with MSK or CNS symptoms. (C) Top 10 enriched gene sets ranked by normalized enrichment scores (NES) associated with both MSK and CNS adverse event (AE) groups. Unique gene sets ranked by NES for subjects with ( $\mathbf{D}$ and $\mathbf{E}$ ) MSK and (F) CNS AEs. Heatmaps of genes in adenosine diphosphate (ADP) signaling, sphingolipid metabolism, anaphase-promoting complex/cyclosome (APC/C) regulation of the cell cycle, activation of prereplicative complex, signaling by stem cell factor (SCF) kit, and SEMA4D in semaphorin signaling for subjects with (G and H) MSK AEs and (I) CNS AEs.

The development of AEs may also not be due to YFLAV but rather other constituents in the vaccine. YFLAV is produced in embryonated chicken eggs and recovered through addition of sterile water, homogenization, and clarification by centrifugation to yield supernatant fluid harvest (35). Following sterility tests, the virus is then suspended in a stabilizing medium containing a mixture of buffers and salts (35). Whether trace levels of egg antigen or any components of the stabilizing medium contribute to the development of AEs cannot be established from our study. Studies that include a placebo produced in the same way as YFLAV but with mock-infected embryonated eggs 
will be needed to dissect among the various components of YFLAV to identify the exact stimulus of the correlates of AEs.

Our findings have another important medical implication. As the median time of AE onset coincides with the expression of the molecular correlates of immunogenicity, administration of antiinflammatory drugs during this time to alleviate AEs could potentially suppress adaptive immunity. Indeed, this outcome has been previously suggested in some clinical studies that observed reduce antibody titers in subjects who received anti-pyretics after vaccination (36). Our findings provide a plausible molecular explanation for this outcome and raise caution on the use of antiinflammatory drugs after vaccination.

In conclusion, we observed no association between previously identified correlates of immunogenicity with development of AEs. Although the innate immune response underlies the development of both immunogenicity and reactogenicity, the temporal separation in the molecular correlates of these outcomes suggests that they are not the inseparable yin-yang of vaccination.

\section{Methods}

Clinical trial design and randomization. The results of this study were based on an exploratory analysis of a recently published, open-label, randomized clinical trial that evaluated the impact of cross-reactive Japanese encephalitis (JE) antibodies on YF vaccine immunogenicity conducted at the SingHealth Investigational Medicine Unit in Singapore (5). The study was initiated in 2013 and completed by 2015. The design of this study and statistical methods were previously described in detail (5). In brief, volunteers were eligible if they were prescreened to be negative for anti-dengue antibodies by ELISA (Panbio Dengue, IgG, Alere), had not previously received JE and YF vaccinations, had normal baseline serum hematological/renal/hepatic chemistry levels, and no contraindications against immunization (immunosuppression, pregnancy, known hypersensitivity reaction to vaccine components). These subjects were randomized into 2 arms: subjects in the JE vaccine arm received 2 doses of inactivated JE vaccine (Ixiaro, Novartis Singapore Pte Ltd) followed by YFLAV administration (Stamaril, Sanofi Pasteur) at 1, 4, or 9 months after JE vaccination; subjects in the YFLAV-only arm received no prior JE vaccination. With the exception of one subject who was recruited last in the clinical trial, all subjects received the same YFLAV batch. Serum was collected on days 0 (pre-YFLAV administration), 1, 3, 7, and 1 month after vaccination for analysis. Throughout the entire study period, all local and systemic symptoms experienced up to 1 month after JE and YF vaccination were collected. For this study, only symptoms reported following YFLAV administration were analyzed.

Safety and AE assessment. Assessment of safety and AEs following YFLAV administration was previously described in detail (5). In brief, an AE following immunization is defined as any untoward medical occurrence that follows immunization and that does not necessarily have a causal relationship with the vaccine's usage. A serious AE is defined as any untoward medical occurrence that is life-threatening, requires in-patient hospitalization, results in persistent or significant disability, or is a congenital defect. Participants were educated to use a daily diary to record any local and systemic symptoms for a 1-month period following immunization. During each study visit after YFLAV administration on days 1, 3, 7, 15, and 28, the participant underwent physical examination, vital signs measurement (blood pressure, temperature, pulse rate, and respiratory rate), and diary review by a team of trained physicians. The reported AEs were described in accordance with CTCAE version 4.03 recommendations (16). Causality was determined by the evaluating physician based on the temporal relationship of AEs to administration of YFLAV, clinical judgment, and the evidence of signs and symptoms indicating alternative diagnoses. Each AE terminology, the time and date of event start and end, severity (1, mild; 2 , moderate; 3 , severe), relatedness to vaccination (1, not related; 2 , unlikely related; 3 , possibly related; 4 , probably related; 5 , definitely related), treatment given, and outcome of the event was recorded from day 0 to month 1 following YFLAV administration or until resolution of the event. Although all AEs were noted, only AEs of which symptoms considered possibly, probably, or definitely related to YFLAV administration not limited to local injection site (including vaccination site pain, bruising, redness, itch, and swelling) were further analyzed in this study. Using the distribution of fever, which began on day 1 after vaccination as a guide, all subjects with systemic AEs were divided into (a) the immediate group if they reported AEs within 24 hours after vaccination; or (b) the delayed group if they either reported immediate AEs within 24 hours of vaccination that resolved before reports of new onset AEs after 24 hours after vaccination, or reported AEs only after 24 hours after vaccination. All AEs were further classified into organ system classes based on CTCAE definitions (16): CNS, MSK, respiratory (RESP), and gastrointestinal (GI) AEs. Only the most common organic-specific AEs - CNS and MSK - could be further analyzed in this study. 
Viruses. YF17D virus was isolated from Stamaril, passaged once in Vero cells (ATCC) and stored at $-80^{\circ} \mathrm{C}$ until use. Infectious titer was quantified by plaque assay as previously described (5). All cells were tested to be free from mycoplasma contamination.

Plaque reduction neutralization test. Plaque reduction neutralization test (PRNT) was performed on BHK-21 cells (ATCC) as previously described using test subjects' sera obtained on day 0 and 1 month after YFLAV administration (5). All cells were tested to be free from mycoplasma contamination. Briefly, serial 2-fold dilution of sera in RPMI maintenance media (MM) was incubated with 40 PFU of YF17D virus in equal volumes $(100 \mu \mathrm{l})$ for 1 hour followed by addition to BHK-21 cells. The mixture was aspirated after incubation for 1 hour at $37^{\circ} \mathrm{C}$, and cells were overlaid with $1 \%$ carboxymethyl cellulose in MM. After incubation for 5 days at $37^{\circ} \mathrm{C}$, cells were fixed with the addition of $20 \%$ formaldehyde and stained with $1 \%$ crystal violet. PRNT $_{50}$ values were determined with a sigmoid dose-response curve fit and reported as reciprocal values. For each subject, internal controls were available by collecting serum samples before and after vaccination for determination of neutralization-antibody titer. All experiments were conducted in technical triplicates.

$Y F 17 D$ viremia assessment with $q P C R$. Viral RNA from sera was extracted using a viral RNA isolation kit (Qiagen), followed by cDNA synthesis (iScript, Bio-Rad) and real-time qPCR (SYBR Green, Roche) in accordance with the manufacturer's protocol. The YF17D primers, RNA copy number standards used, and calculation methods were published previously (5). All experiments were conducted in technical triplicates.

Gene expression analysis with microarray. Whole blood was collected from volunteers on days 0,1 , and 3 in relation to YFLAV administration and stored in Tempus blood RNA tubes at $-80^{\circ} \mathrm{C}$. RNA isolation and DNAse treatment were performed according to the manufacturer's protocol (PAXgene blood RNA kit, Qiagen). Microarray was performed with Illumina Human HT-12 v4 BeadChips at the Duke-NUS Genome Biology Core Facility as previously described and deposited in the ArrayExpress database (www.ebi.ac.uk/arrayexpress) under accession number E-MTAB-4669 (5). Each experiment was performed in technical duplicates.

GSEA. Analysis of microarray data was performed using Partek software as described previously (5). Enrichment for up- or downregulated sets of genes from the REACTOME pathway database (version 5.2) was computed by running GSEA (GSEA Java software version 2.2.3, Broad Institute) against the fold change of day 1 or day 3 over day 0 (baseline) samples in subjects with either immediate or delayed AEs with those without AEs (17). Gene sets were considered to be significantly enriched if their NES had an FDR $q$ value of less than 0.25 , as previously described (17). Genes within a gene set that accounts for the enrichment score computed by GSEA (termed the leading-edge subset) were selected for generation of heatmaps and further validation with nCounter (17). Heatmaps were created using GENE-E (Broad Institute, available on https://software.broadinstitute.org/GENE-E/).

$n$ Counter RNA measurement. For nCounter (Nanostring Technologies) validation of microarray findings, RNA was isolated from volunteers' whole blood collected on days 0 and 1 in relation to YFLAV administration were used, and concentrations measured by RiboGreen (Thermo Fisher Scientific) according to the manufacturer's instructions. A custom-designed gene expression probe set was used. Total RNA (200 ng) was hybridized to the reporter and capture probe sets at $65^{\circ} \mathrm{C}$ for 12 hours. Hybridized complexes were immobilized on the nCounter Prep Station (Nanostring Technologies) using the high-sensitivity protocol and scanned using the nCounter Digital Analyzer (Nanostring Technologies) at 555 fields of view (FOV). Each experiment was performed in technical duplicates.

Data from nCounter were analyzed using NanoString's nSolver analysis software (version 3.0) as per the manufacturer's protocols. Internal negative control probes included in each assay were used to determine the background threshold (2 SD above the mean negative control probe count value) for each sample. Positive control count values were then used to normalize samples for any differences in sample preparation, hybridization, and nCounter Prep Station processing efficiency. No nCounter samples were flagged for quality control. Counts were normalized using 6 included housekeeping genes (TBP, ACTB, B2M, $D E C R 1, P G K 1$, and $P P I B)$. Changes in gene expression on day 0 versus day 1 in the form of $\log 2$ ratio and fold change were calculated for each subject.

Identification of autism spectrum disorder (ASD) genes. Genes linked to ASD were classified into 6 categories by AutDB (https://gene.sfari.org/autdb/GS_Home.do) (accessed 23 Feb 2017) (31): category S (syndromic genes), category 1 (high confidence genes), category 2 (strong candidate genes), category 3 (suggestive evidence genes), category 4 (minimal evidence genes), category 4 (hypothesized genes), and category 6 (not supported genes). All genes from category S, 1, 2, and 3 were compared with genes enriched in individuals with AEs. 
Statistics. Data are represented as mean \pm SEM. Statistical analyses were performed using Prism 6.0 software (GraphPad Software Inc.), and significance was determined by 2-tailed, Mann-Whitney test. $P$ values of less than 0.05 were considered statistically significant. For GSEA data, gene sets were considered to be significantly enriched when their NES had an FDR $q$ value of less than 0.25 . Pearson's correlation $(r)$ analysis of nCounter data was performed using RStudio 1.0.143 (RStudio, Inc), and $P$ values of less than 0.05 were considered statistically significant.

Study approval. In brief, the study was approved by the SingHealth Centralized Institutional Review Board (ID: 2013/385/E) and was carried out in accordance with the principles of the Singapore Good Clinical Practice guidelines and in compliance with the Helsinki Declaration. The trial was registered on clinicaltrial.gov (registration number: NCT01943305). Informed written consent was obtained from all participants before inclusion in the study.

Supplemental data. Any supplemental information and data files are available in the online version of the paper.

\section{Author contributions}

JGL and EEO conceived the study. KRC conducted the microarray, viremia, and neutralizing antibody experiments. CYYC designed the nCounter experiments and analyzed the gene expression data. CJHC and SH supported the nCounter experiments. SG analyzed the microarray data. CYYC, KRC, EEO, and JGL wrote the manuscript.

\section{Acknowledgments}

We thank X.R. Chai for her computational support. This study was funded by the Biomedical Research Council of Singapore, under the Translational Clinical Research Program grant (13/1/96/19/689). C.Y.Y. Chan is supported by the National Medical Research Council of Singapore (NMRC) (MH095:003/008-194). C.J.H. Chua and S. Hazirah are supported in STIIC with grants from NMRC (NMRC/STaR/020/2013, NMRC/ MOHIAFCAT2/005/2015, MOHIAFCAT2001, CIRG13nov032, and NMRC MOHIAFCAT1-6003). J.G. Low is supported by a Clinician-Scientist Award from the NMRC (NMRC/CSA-INV/0013/2016). E.E. Ooi is supported by a Clinician-Scientist Award from the NMRC (NMRC/CSA/060/2014).

Address correspondence to: Eng Eong Ooi, Emerging Infectious Diseases, Duke-NUS Medical School, 8 College Road, Singapore 169857. Phone: 65.6516.8594; Email: engeong.ooi@duke-nus.edu.sg. Or to: Jenny G. Low, Department of Infectious Diseases, Singapore General Hospital, 20 College Road, Singapore 169856. Phone: 65.6321.3479; Email: jenny.low@singhealth.com.sg.

1. Doherty M, Buchy P, Standaert B, Giaquinto C, Prado-Cohrs D. Vaccine impact: benefits for human health. Vaccine 2016;34(52):6707-6714.

2. Whitaker JA, Ovsyannikova IG, Poland GA. Adversomics: a new paradigm for vaccine safety and design. Expert Rev Vaccines. 2015;14(7):935-947.

3. Gaucher D, et al. Yellow fever vaccine induces integrated multilineage and polyfunctional immune responses. J Exp Med. 2008;205(13):3119-3131.

4. Querec TD, et al. Systems biology approach predicts immunogenicity of the yellow fever vaccine in humans. Nat Immunol. 2009;10(1):116-125.

5. Chan KR, et al. Cross-reactive antibodies enhance live attenuated virus infection for increased immunogenicity [published online ahead of print September 19, 2016]. Nat Microbiol. doi: 10.1038/nmicrobiol.2016.164.

6. Hou J, et al. A systems vaccinology approach reveals temporal transcriptomic changes of immune responses to the yellow fever 17D vaccine. J Immunol. 2017;199(4):1476-1489.

7. Pulendran B. Learning immunology from the yellow fever vaccine: innate immunity to systems vaccinology. Nat Rev Immunol. 2009;9(10):741-747.

8. Takeuchi O, Akira S. Pattern recognition receptors and inflammation. Cell. 2010;140(6):805-820.

9. Dantzer R, O’Connor JC, Freund GG, Johnson RW, Kelley KW. From inflammation to sickness and depression: when the immune system subjugates the brain. Nat Rev Neurosci. 2008;9(1):46-56.

10. Galen JE, Curtiss R. The delicate balance in genetically engineering live vaccines. Vaccine. 2014;32(35):4376-4385.

11. Camacho LA, et al. Reactogenicity of yellow fever vaccines in a randomized, placebo-controlled trial. Rev Saude Publica. 2005;39(3):413-420.

12. Monath TP, et al. Comparative safety and immunogenicity of two yellow fever 17D vaccines (ARILVAX and YF-VAX) in a phase III multicenter, double-blind clinical trial. Am J Trop Med Hyg. 2002;66(5):533-541.

13. Lang J, Zuckerman J, Clarke P, Barrett P, Kirkpatrick C, Blondeau C. Comparison of the immunogenicity and safety of two 17D yellow fever vaccines. Am J Trop Med Hyg. 1999;60(6):1045-1050. 
14. Ripoll C, et al. Evaluation of two yellow fever vaccines for routine immunization programs in Argentina. Hum Vaccin. 2008;4(2):121-126.

15. Pfister M, et al. Immunogenicity and safety of BERNA-YF compared with two other 17D yellow fever vaccines in a phase 3 clinical trial. Am J Trop Med Hyg. 2005;72(3):339-346.

16. National Cancer Institute. Common Terminology Criteria for Adverse Events version 4.03 (CTCAE). US Department of Health and Human Services, National Institute of Health, National Cancer Institute; 2010. https://evs.nci.nih.gov/ftp1/CTCAE/ CTCAE_4.03_2010-06-14_QuickReference_5x7.pdf. NCI Website. Published May 28, 2009. Updated June 14, 2010. Accessed September 4, 2017.

17. Subramanian A, et al. Gene set enrichment analysis: a knowledge-based approach for interpreting genome-wide expression profiles. Proc Natl Acad Sci USA. 2005;102(43):15545-15550.

18. Payton JE, et al. High throughput digital quantification of mRNA abundance in primary human acute myeloid leukemia samples. J Clin Invest. 2009;119(6):1714-1726.

19. Ren K, Dubner R. Interactions between the immune andnervous systems in pain. Nat Med. 2010;16(11):1267-1276

20. McMahon SB, La Russa F, Bennett DL. Crosstalk between the nociceptive and immune systems in host defence and disease. Nat Rev Neurosci. 2015;16(7):389-402.

21. Cekic C, Linden J. Purinergic regulation of the immune system. Nat Rev Immunol. 2016;16(3):177-192.

22. Burnstock G. Purinergic mechanisms and pain. Adv Pharmacol. 2016;75:91-137.

23. Germain A, Ruppert D, Levine SM, Hanson MR. Metabolic profiling of a myalgic encephalomyelitis/chronic fatigue syndrome discovery cohort reveals disturbances in fatty acid and lipid metabolism. Mol Biosyst. 2017;13(2):371-379.

24. Light AR, White AT, Hughen RW, Light KC. Moderate exercise increases expression for sensory, adrenergic, and immune genes in chronic fatigue syndrome patients but not in normal subjects. J Pain. 2009;10(10):1099-1112.

25. Gow JW, Hagan S, Herzyk P, Cannon C, Behan PO, Chaudhuri A. A gene signature for post-infectious chronic fatigue syndrome. BMC Med Genomics. 2009;2:38.

26. Whistler T, Unger ER, Nisenbaum R, Vernon SD. Integration of gene expression, clinical, and epidemiologic data to characterize chronic fatigue syndrome. J Transl Med. 2003;1(1):10.

27. Ray P, Krishnamoorthy N, Oriss TB, Ray A. Signaling of c-kit in dendritic cells influences adaptive immunity. Ann N Y Acad Sci. 2010;1183:104-122.

28. Milenkovic N, et al. Nociceptive tuning by stem cell factor/c-Kit signaling. Neuron. 2007;56(5):893-906

29. Jensen BM, Akin C, Gilfillan AM. Pharmacological targeting of the KIT growth factor receptor: a therapeutic consideration for mast cell disorders. Br J Pharmacol. 2008;154(8):1572-1582.

30. Worzfeld T, Offermanns S. Semaphorins and plexins as therapeutic targets. Nat Rev Drug Discov. 2014;13(8):603-621.

31. Basu SN, Kollu R, Banerjee-Basu S. AutDB: a gene reference resource for autism research. Nucleic Acids Res. 2009;37(Database issue):D832-D836.

32. Kioussis D, Pachnis V. Immune and nervous systems: more than just a superficial similarity? Immunity. 2009;31(5):705-710.

33. Vaughn DW, et al. Dengue viremia titer, antibody response pattern, and virus serotype correlate with disease severity. $J$ Infect Dis. 2000;181(1):2-9.

34. Chau TN, et al. Clinical and virological features of Dengue in Vietnamese infants. PLoS Negl Trop Dis. 2010;4(4):e657.

35. Monath TP, Gershman M, Staples JE, Barrett ADT. Yellow fever vaccine. In: Plotkin S, Orenstein W, Offit P, eds. Vaccines. Philadelphia, Pennsylvania, USA: Elsevier, Inc.; 2013:870-968.

36. Prymula R, et al. Effect of prophylactic paracetamol administration at time of vaccination on febrile reactions and antibody responses in children: two open-label, randomised controlled trials. Lancet. 2009;374(9698):1339-1350. 